\title{
Performances pondérales de la pintade locale (Numida meleagris) en système d'alimentation améliorée dans la zone centrale du Burkina Faso
}

\author{
R. Sanfo ${ }^{1 *}$ H. Boly ${ }^{2}$ L. Sawadogo ${ }^{3}$ O. Brian ${ }^{4}$
}

Mots-clés

Pintade - Numida meleagris Performance animale - Gain de poids - Burkina Faso.

\begin{abstract}
Résumé
Les performances de croissance pondérale de 200 pintades (97 femelles et 103 mâles) locales (Numida meleagris) en système d'alimentation améliorée ont été rapportées. Les femelles ont présenté un poids vif moyen à l'éclosion de 25,8 $\pm 3,1 \mathrm{~g}$ et les mâles de 25,5 $\pm 2,4 \mathrm{~g}$. Les poids vifs aux points d'inflexion ont été de 523,0 $\pm 171,4 \mathrm{~g}$ chez les femelles et de 497,0 $\pm 134,7 \mathrm{~g}$ chez les mâles, et les poids asymptotiques respectivement de $962,0 \pm 147,8 \mathrm{~g}$ et $857,0 \pm 183,7 \mathrm{~g}$. Les poids aux points d'inflexion et les poids asymptotiques ont différé significativement $(p<0,05)$ selon la variété. Les gains de poids moyens quotidiens aux points d'inflexion ont été de 10,1 $\pm 4,7 \mathrm{~g}$ chez les femelles et de 10,2 $\pm 5,1$ g chez les mâles. Ils ont été acquis dans les intervalles d'âges respectifs de 12 à 14 semaines et 14 à 16 semaines, âges optimums à l'abattage. La moyenne générale des taux de croissance a été de 29,2 $\pm 16,8$ p. 100 chez les deux sexes, avec $30,0 \pm 18,0$ p. 100 chez les femelles et $28,4 \pm 15,9$ p. 100 chez les mâles. Le rendement carcasse a augmenté de 65,7 p. 100 à 16 semaines à 74,3 p. 100 à 22 semaines, pour baisser à 70,4 p. 100 à 56 semaines. L'indice moyen de consommation a présenté la valeur de 4,6 de l'éclosion à 16 semaines d'âge chez les deux sexes.
\end{abstract}

\section{INTRODUCTION}

L'élevage de la pintade locale (Numida meleagris) connaît un intérêt de plus en plus soutenu dans le secteur du développement rural au Burkina Faso (6). Cette espèce représente 20 p. 100 de l'effectif avicole national, estimé à près de 31000000 sujets (23), et constitue une source importante et facilement mobilisable de protéines animales et de revenus pour de nombreuses communautés (24). Elle offre

1. Inera / Kamboinsé, département des Productions animales, Ouagadougou, Burkina Faso.

2. Inera, Ouagadougou, Burkina Faso.

3. Université d'Ouagadougou, Burkina Faso.

4. University of Agricultural Sciences, Department of Animal Nutrition and Management, Uppsala, Sweden.

* Auteur pour la correspondance

Inera / Kamboinsé, département des Productions animales, 01 BP 476, Ouagadougou 01, Burkina Faso.

Tél. : +22650319229; e-mail : r_sanfo@yahoo.fr de grandes opportunités de développement à travers ses productions de viande et d'œufs, sa rusticité et sa bonne répartition géographique au plan national $(12,13)$.

En dépit de cette importance et contrairement aux ruminants, la pintade locale n'a pas fait l'objet d'un intérêt particulier, tant de la part des techniciens du développement rural que de la recherche (28). Son système d'élevage relève plus de la cueillette, avec une alimentation essentiellement sur parcours libres, des abris sommaires en guise d'habitat et une couverture sanitaire presque inexistante (29). Ce désintérêt relatif expliquerait les faibles performances de production reconnues à la pintade locale (28).

L'objectif de cette étude a été de déterminer le potentiel de croissance de la pintade locale dans des conditions d'habitat, d'alimentation et de santé améliorées. Il s'articule autour de trois axes : déterminer les performances de croissance pondérale, estimer l'âge optimal à l'abattage, et déterminer les indices de consommation et les rendements carcasse à âge types. 


\section{MATERIEL ET METHODES}

Les animaux de cette étude, 97 femelles et 103 mâles, tirés d'une population de 772 pintadeaux, ont été obtenus suite à une incubation artificielle d'œufs de pintades locales. Les œufs ont été récoltés auprès de cinq producteurs du village de Saria, dans la région centrale du Burkina Faso, sur la base de l'effectif du cheptel. Un total de 2500 œufs ont été incubés et le taux d'éclosion a été de 65,7 p. 100 avec un taux de mortalité de 9,1 p. 100. Les pintadeaux retenus ont été identifiés par des boucles alaires, gardés dans des éleveuses locales de type Attesta, et chauffés à l'aide de lampes à pétrole à une température moyenne de $37^{\circ} \mathrm{C}$ jusqu'à l'âge de huit semaines. Ils ont ensuite été transférés dans des habitats pour adultes. A 20 semaines d'âge, un sexage basé sur la forme et la consistance des éminences génitales (23) a été effectué.

Les pesées, réalisées après un jeûne de 12 heures, ont été effectuées toutes les deux semaines à partir de l'éclosion jusqu'à 24 semaines, avec une balance électrique d'une précision de $0,1 \mathrm{mg}$ jusqu'à huit semaines, puis poursuivies avec une balance électronique d'une précision de $5 \mathrm{~g}$. Une observation ponctuelle du poids a été effectuée à 56 semaines d'âge pour calculer les rendements carcasse.

Les sujets de l'étude ont été nourris avec des aliments industriels distribués ad libitum selon trois types de régime :

- démarrage de 0 à 4 semaines ; protéines brutes $(\mathrm{PB})=$ $19,8 \mathrm{~g} / 100 \mathrm{~g}$ de matière sèche $(\mathrm{MS})$; énergie brute $(\mathrm{EB})=$ $2970 \mathrm{kcal} / \mathrm{g}$ de MS ;

- croissance de 5 à 8 semaines ; PB = 16,5 g / $100 \mathrm{~g}$ de MS ; $\mathrm{EB}=2900 \mathrm{kcal} / \mathrm{g}$ de $\mathrm{MS}$ ) ;

- finition de 8 à 24 semaines ; $\mathrm{PB}=17 \mathrm{~g} / 100 \mathrm{~g}$ de $\mathrm{MS}$; $\mathrm{EB}=2750 \mathrm{kcal} / \mathrm{g}$ de $\mathrm{MS}$.

Les consommations alimentaires ont été mesurées par la pesée quotidienne des quantités distribuées et refusées. L'eau de boisson a été apportée à volonté avec du saccharose dissout à 2,6 p. 100 pour permettre aux pintadeaux d'accentuer la lutte contre le froid (10).

La prophylaxie médicale a consisté en une vaccination contre la maladie de Newcastle, au $21^{\mathrm{e}}$ jour après l'éclosion et un rappel tous les six mois. Par la suite, les autres vaccinations ont été précédées de déparasitages internes à l'aide de trichonomonacide ou vermifuge spécial pintade (VSP). Les différents phénotypes de pintades ont été distingués sur la base de la couleur grise, noire, blanche et lilas du plumage $(8,14)$.

Le taux de croissance a été calculé selon la formule de MignonGrasteau et Beaumont (21) :

$\mathrm{T}=\left[\left(\mathrm{W}_{2}-\mathrm{W}_{1}\right) /\left(\mathrm{W}_{2}+\mathrm{W}_{1}\right) / 2\right] \times 100$

où $\mathrm{T}$ est le taux de croissance $(\%), \mathrm{W}_{1}$ le poids initial, $\mathrm{W}_{2}$ le poids final.

Les paramètres de la courbe de croissance ont été estimés selon la régression non linéaire dont la formule est $(17,21)$ :

$\mathrm{Pt}=\mathrm{P}_{0} \mathrm{eT}^{(1-\mathrm{e}-\mathrm{Dt}) / \mathrm{D}}$

où Pt est le poids au temps $\mathrm{t}, \mathrm{P}_{0}$ le poids à $(\mathrm{t}=0)$, $\mathrm{T}$ le taux spécifique de croissance, $\mathrm{D}$ le paramètre de déclin ou taux de maturité.

Le temps au point d'inflexion $\left(\mathrm{T}_{\mathrm{I}}\right)$ et le poids asymptotique $\left(\mathrm{P}_{\mathrm{A}}\right)$ ont été estimés par les formules de Knizetova et coll., et MignonGrasteau et Beaumont $(17,21)$. $\mathrm{T}_{\mathrm{I}}$ est l'âge où la croissance est maximale. Il correspond à l'âge d'abattage, c'est-à-dire l'âge où le gain de poids moyen quotidien (gmq) est maximal. $\mathrm{P}_{\mathrm{A}}$ est le poids quand l'âge tend vers l'infini. La mise en œuvre de ce modèle a respecté l'application de la moyenne des points. Les rendements carcasse ont été calculés sur 50 pintadeaux retenus par un tirage aléatoire de dix sujets (cinq femelles et cinq mâles) par âge type à
16, 18, 20, 22 et 56 semaines. Les abattages ont été effectués après une diète hydrique de 12 heures.

Les données recueillies ont été analysées à l'aide du logiciel StatView. Les comparaisons des moyennes ont été faites par le test $\mathrm{t}$ de Student au seuil de signification de 5 p. 100.

\section{RESULTATS}

\section{Taux d'éclosion et de mortalité}

Le taux de fertilité des œufs achetés aux producteurs a été de 89,8 p. 100, le taux d'éclosion de 65,7 p. 100, le taux de mortalité embryonnaire de 34,3 p. 100, avec un taux de mortalité précoce de 27,0 p. 100 et un taux de mortalité à l'éclosion de 7,3 p. 100. Le taux de mortalité de 9,1 p. 100 a été enregistré chez les pintadeaux soumis à l'expérience. Les mortalités sont survenues généralement la nuit sans aucun signe pathologique apparent.

\section{Poids vifs selon le sexe}

L'évolution pondérale selon l'âge est indiquée dans la figure 1. L'évolution de la courbe de croissance a connu une régularité avec le temps.

\section{Poids vifs selon le phénotype}

Dans le tableau I sont inscrits les poids vifs à l'éclosion, au point d'inflexion, et les poids asymptotiques selon le phénotype. Aucune différence significative n'a été mise en évidence quant au $\mathrm{P}_{0}$ selon le phénotype.

\section{Gains moyens quotidiens}

L'évolution des gmq selon le sexe est représentée à la figure 2. Chaque courbe évolutive a été marquée par trois phases :

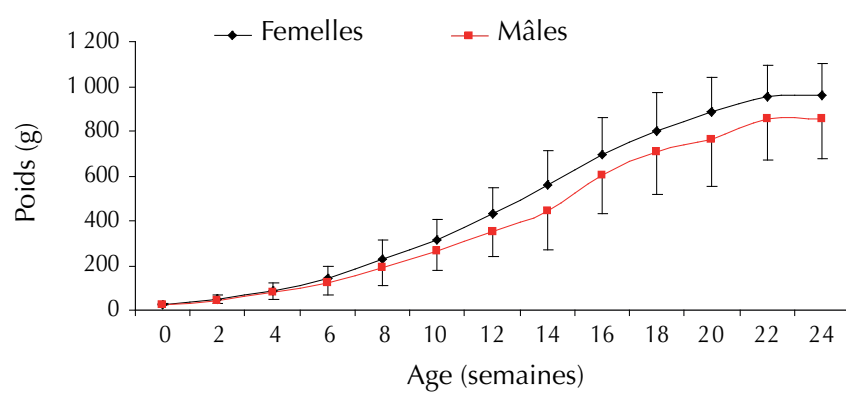

Figure 1 : courbe de l'évolution pondérale selon le sexe.

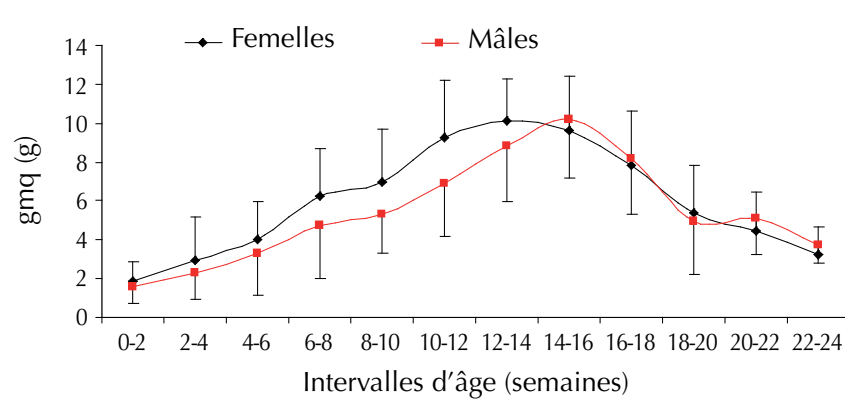

Figure 2 : courbes évolutives des gains de poids moyens quotidiens selon le sexe. 


\section{Tableau}

Poids vifs à l'éclosion, au point d'inflexion et asymptotique selon le type phénotypique

\begin{tabular}{lcccc} 
Variété & Lilas $(\mathbf{n}=\mathbf{1 0})$ & Noire $(\mathbf{n}=\mathbf{1 8})$ & Perlée $(\mathbf{n}=\mathbf{1 4 2})$ & Blanche $(\mathbf{n}=\mathbf{3 0})$ \\
\hline$P_{0}$ (g) & $25,7 \pm 1,7^{\mathrm{a}}$ & $26,5 \pm 2,4^{\mathrm{a}}$ & $25,5 \pm 2,7^{\mathrm{a}}$ & $23,0 \pm 3,5^{\mathrm{a}}$ \\
$\mathrm{P}_{1}(\mathrm{~g})$ & $601,7 \pm 163,1^{\mathrm{a}}$ & $757,5 \pm 107,1^{\mathrm{b}}$ & $662,3 \pm 165,3^{\mathrm{c}}$ & $634,0 \pm 170,3^{\mathrm{d}}$ \\
$\mathrm{P}_{\mathrm{A}}$ (g) & $902,6 \pm 115,4^{\mathrm{a}}$ & $1001,3 \pm 215,1^{\mathrm{b}}$ & $961,1 \pm 215,1^{\mathrm{c}}$ & $924,7 \pm 127,5^{\mathrm{d}}$
\end{tabular}

$\mathrm{P}_{0}$ : poids à l'éclosion; $\mathrm{P}_{\mathrm{I}}$ : poids au point d'inflexion $; \mathrm{P}_{\mathrm{A}}$ : poids asymptotique

Les chiffres présentant les mêmes lettres sur une ligne ne diffèrent pas significativement au seuil de $5 \%$

ascendante (de $\mathrm{P}_{0}$ à $\left.\mathrm{P}_{\mathrm{I}}\right)$, en plateau (à $\left.\mathrm{P}_{\mathrm{I}}\right)$ et descendante $\left(\mathrm{P}_{\mathrm{I}}\right.$ à $\left.\mathrm{P}_{\mathrm{A}}\right)$. De $\mathrm{P}_{0}$ à $\mathrm{P}_{\mathrm{A}}$, le gmq moyen a été de $5,9 \pm 2,8 \mathrm{~g}$ pour l'ensemble des animaux $(6,3 \pm 2,8 \mathrm{~g}$ pour les femelles et $5,4 \pm 2,7 \mathrm{~g}$ pour les mâles). Le gmq moyen au point d'inflexion a été de 10,2 $\pm 4,9 \mathrm{~g}$ (10,1 $\pm 4,7 \mathrm{~g}$ chez les femelles et $10,2 \pm 5,1 \mathrm{~g}$ chez les mâles). Les $\mathrm{gmq}_{\mathrm{P} 0 \text {-PI }}$ ont été de $5,8 \pm 3,2 \mathrm{~g}$ chez les femelles et de $5,4 \pm 2,9 \mathrm{~g}$ chez les mâles.

\section{Taux de croissance}

Les taux de croissance pondérale, ou les paramètres de déclin ou taux de maturité, selon les stades de développement (démarrage, croissance, finition) sont présentés dans le tableau II. Chez les deux sexes, les taux moyens de croissance ont marqué une évolution à la baisse avec l'âge de l'animal. Chez les femelles, cette baisse a été en moyenne de 5,3 p. 100 entre deux intervalles successifs de deux semaines contre 4,5 p. 100 chez les mâles. Le taux de croissance moyen a été de $29,2 \pm 16,8$ p. $100 \mathrm{chez}$ les deux sexes $(30,0 \pm$ 18,0 p. 100 chez les femelles et $28,4 \pm 15,9$ p. 100 chez les mâles). La différence entre les deux sexes a été significative au seuil de 5 p. 100.

\section{Résultats de la modélisation}

Les données du poids vif, de $\mathrm{P}_{0}$ à $\mathrm{P}_{\mathrm{A}}$, sont inscrites dans le tableau III. Aucune différence significative ( $p>0,05)$ n'a été observée pour le poids vif à l'éclosion $\left(\mathrm{P}_{0}\right)$ selon le sexe. A deux semaines d'âge, les pintadeaux ont présenté un poids moyen de $47,9 \pm 1,9 \mathrm{~g}$, avec $50,1 \pm 2,5 \mathrm{~g}$ pour les femelles, $45,7 \pm 1,3 \mathrm{~g}$ pour les mâles. A partir des poids aux points d'inflexion $\left(\mathrm{P}_{\mathrm{I}}\right)$, les poids vifs ont marqué des différences significatives $(\mathrm{p}<0,05)$ selon le sexe, de même que les poids asymptotiques $\left(\mathrm{P}_{\mathrm{A}}\right)$. Le poids vif aux points d'inflexion $\left(\mathrm{P}_{\mathrm{I}}\right)$ a représenté plus de la moitié du poids asymptotique $\left(\mathrm{P}_{\mathrm{A}}\right)$ chez les deux sexes $(54,4 \pm 1,2$ p. 100 chez les femelles et $58,0 \pm$ 0,7 p. 100 chez les mâles). Les poids aux points d'inflexion $\left(\mathrm{P}_{\mathrm{I}}\right)$ et les poids asymptotiques $\left(\mathrm{P}_{\mathrm{A}}\right)$ ont, en revanche, été significativement $(\mathrm{p}<0,05)$ affectés par le sexe.

Il a été observé un décalage entre les points d'inflexion selon le sexe (dans l'intervalle de 12 à 14 semaines chez les femelles et de 14 à 16 chez les mâles). Les poids asymptotiques ont été atteints autour de la $22^{\mathrm{e}}$ semaine chez les deux sexes.

\section{Indices de consommation}

Les données sur l'alimentation et les gains de poids relatifs sont notés dans le tableau IV. L'indice moyen de consommation a augmenté avec l'âge de l'animal. Il a été en moyenne de 4,6 du démarrage (0-4 semaines) à la finition (8-24 semaines).

\section{Tableau II}

Taux de croissance, ou paramètre D, selon le stade de développement

\begin{tabular}{lcccc}
$\begin{array}{l}\text { Stade de } \\
\text { développement }\end{array}$ & Démarrage & Croissance & \multicolumn{2}{c}{ Finition } \\
$\begin{array}{l}\text { Intervalle d'âge } \\
\begin{array}{l}\text { (semaine) } \\
\text { Taux de croissance (\%) }\end{array}\end{array}$ & $0-4$ & $5-8$ & $9-16$ & $17-22$ \\
$\quad$ Femelle & 55,9 & 45,2 & 30,4 & 10,6 \\
$\quad$ Mâle & 49,5 & 42,7 & 29,0 & 11,6 \\
& & & & \\
\end{tabular}

Tableau III

Résultats de la modélisation

\begin{tabular}{lccc} 
& $\mathbf{P}_{\mathbf{0}}(\mathbf{g})$ & $\mathbf{P}_{\mathbf{I}}(\mathbf{g})$ & $\mathbf{P}_{\mathbf{A}}(\mathbf{g})$ \\
\hline Mâle $(\mathrm{n}=103)$ & $25,5 \pm 2,4^{\text {a }}$ & $497,0 \pm 134,7^{\text {a }}$ & $857,0 \pm 183,7^{\text {a }}$ \\
Femelle $(\mathrm{n}=97)$ & $25,8 \pm 3,1^{\text {a }}$ & $523,0 \pm 171,4^{\mathrm{b}}$ & $962,0 \pm 147,8^{b}$ \\
Moyenne & $25,7 \pm 2,8$ & $510,0 \pm 153,1$ & $909,5 \pm 165,8$
\end{tabular}

$\mathrm{P}_{0}$ : poids à l'éclosion $; \mathrm{P}_{\mathrm{I}}$ : poids au point d'inflexion $; \mathrm{P}_{\mathrm{A}}$ : poids asymptotique Les chiffres présentant les mêmes lettres en exposant dans une colonne ne diffèrent pas significativement au seuil de $5 \%$

Tableau IV

Données globales sur l'alimentation

\begin{tabular}{|c|c|c|c|c|}
\hline $\begin{array}{l}\text { Intervalle d'âge } \\
\text { (semaine) }\end{array}$ & $0-4$ & $0-8$ & $0-16$ & $0-24$ \\
\hline $\begin{array}{l}\text { Indice de } \\
\text { consommation }\end{array}$ & 2,3 & 4,7 & 6,9 & 12,7 \\
\hline $\begin{array}{l}\text { Consommation (kg) } \\
\text { aliment brut }\end{array}$ & 0,55 & 2,40 & 4,6 & 12,4 \\
\hline Gain de poids (g) & $\begin{array}{l}239,1^{a} \\
\pm 87,6\end{array}$ & $\begin{array}{l}510,6^{b} \\
\pm 101,7\end{array}$ & $\begin{array}{r}666,7^{c} \\
\pm 117,7\end{array}$ & $\begin{array}{l}976,4^{d} \\
\pm 125,5\end{array}$ \\
\hline
\end{tabular}

Les chiffres présentant les mêmes lettres sur une ligne ne diffèrent pas significativement au seuil de $5 \%$ 


\section{Rendements carcasse}

Les donnés bouchères (poids vifs, poids carcasse et rendement carcasse) selon les âges types et le sexe sont indiquées dans le tableau $\mathrm{V}$. Les rendements carcasse ont différé significativement $(\mathrm{p}<0,05)$ d'un âge type à l'autre à l'exception de ceux à 20 et 56 semaines. Ces rendements ont augmenté de la $16^{\mathrm{e}}\left(75,7 \mathrm{p}\right.$. 100) à la $22^{\mathrm{e}}$ semaine (84,3 p. 100), puis ont baissé à la $56^{\mathrm{e}}$ semaine $(80,4$ p. 100).

\section{DISCUSSION}

Les œufs récoltés dans le village de Saria ont eu une durée maximale de stockage de 10 jours. Le taux d'éclosion obtenu a été supérieur à celui de 45 p. 100 relevé par Ayorinde (4) et reste comparable à celui de 58,4 p. 100 rapporté par Sanfo (27) en conditions ambiantes de température et à dix jours de stockage. Le taux de mortalité a été nettement inférieur à celui de 73 p. 100 noté par Bessin et coll. (6) et traduirait l'efficacité du chauffage et de l'apport de sucre dans l'eau de boisson.

L'ascendance relative du poids vif moyen des femelles sur celui des mâles a été conforme aux conclusions de Blum et coll. (7). Selon ces auteurs, qui ont travaillé sur des pintadeaux de chair, la similitude de croissance pondérale entre les deux sexes n'est observée que jusqu'à l'âge de 12 semaines. La maturation sexuelle débutante, qui se manifeste par un dépôt de graisse et le développement des organes génitaux plus marqué chez la femelle, pourrait expliquer cette croissance plus importante.

Le poids vif moyen du pintadeau d'un jour observé dans la présente étude a été comparable à ceux de 27, 26 et 25,2 g rapportés dans la littérature $(8,14,26,30)$. A deux semaines d'âge, ce poids vif s'est révélé inférieur à celui de la variété du Nord avec 63,8 g (8). Cette observation confirmerait les résultats antérieurs qui font cas de l'existence d'une grosse variété de pintade, appelée pintade de Dori et localisée dans la zone sahélienne $(14,26,29)$. Les poids asymptotiques observés chez les deux sexes ont confirmé le caractère « moyen » du poids adulte de la pintade localisée entre les $12^{\mathrm{e}}$ et $13^{\mathrm{e}}$ parallèles $(2,5,16,22)$. Ces poids ont, de même, confirmé la similitude des performances de ces pintades avec celles du Centre dont le poids oscille entre 0,9 et $1,1 \mathrm{~kg}(14,26,29)$.
Les poids vifs à l'éclosion des pintadeaux, selon le phénotype, ont été supérieurs à ceux de la lilas $(23,26 \pm 3,87 \mathrm{~g})$, de la noire $(22,77 \pm 2,98 \mathrm{~g})$, de la perlée $(21,92 \pm 3,65 \mathrm{~g})$ et de la blanche $(20,98 \pm 4,01 \mathrm{~g})$ observés au Nigeria (5). Cette supériorité pourrait résulter d'une différence génétique entre les phénotypes de ces pintades ou du poids des œufs incubés.

Les différences des performances pondérales observées entre les différents phénotypes étaient en accord avec les résultats rapportés au Niger, Nigeria et Burkina Faso (1, 3, 27). Elles étaient également fondamentales et réconfortantes pour la sélection de la pintade pour la viande (4) à cause de l'existence de cette variabilité pondérale.

L'âge au point d'inflexion a coïncidé rigoureusement avec l'âge optimal à l'abattage (19) (donnée technico-économique). Cette correspondance explique l'importance de cet âge (5) et laisse supposer que celui à l'abattage, dans le cas présent, était situé à environ 14 semaines chez les deux sexes. Cet âge est analogue à celui déterminé pour la pintade locale au Nigeria (5). La baisse des gmq, sans effet compensatoire sur la consommation alimentaire au-delà de cet âge $(4,24)$, vient appuyer fortement la nécessité de cette exploitation. Les poids vifs moyens à l'âge optimal à l'abattage des deux sexes ont été supérieurs à la moyenne de 476,6 g observée au Nigeria (3) et représentaient un peu plus de la moitié des poids asymptotiques respectifs.

Le décalage entre les âges aux points d'inflexion chez les deux sexes indique que la croissance pondérale de la pintade locale est liée au sexe et implique qu'une sélection appliquée à âge fixe ne donnera pas la même réponse pour les deux sexes (18). Ainsi, l'âge idéal à la sélection serait de 13 semaines chez les femelles et de 15 semaines chez les mâles.

Les taux moyens de croissance et leur évolution à la baisse avec l'âge ont été comparables à ceux observés pour les pintades locales au Nigeria (3). Les âges aux taux moyens de croissance ont coïncidé avec ceux aux points d'inflexion. Ceci indiquerait qu'il est plus économique d'exploiter la pintade locale à cette période.

La baisse du rendement carcasse à 56 semaines a été analogue à celles de 74 p. 100 à 24 semaines et de 71 p. 100 à 52 semaines observées par Ayorinde (4). Elle pourrait expliquer le fait qu'il est

\section{Tableau V}

Données bouchères selon le sexe et les âges types

\begin{tabular}{|c|c|c|c|c|c|c|c|c|c|}
\hline \multirow[b]{2}{*}{$\begin{array}{l}\text { Age type } \\
\text { (semaines) }\end{array}$} & \multicolumn{3}{|c|}{ Femelles } & \multicolumn{3}{|c|}{ Mâles } & \multicolumn{3}{|c|}{ Moyenne } \\
\hline & $\begin{array}{c}\text { Poids vif } \\
\text { (g) }\end{array}$ & $\begin{array}{c}\text { Poids } \\
\text { carcasse (g) }\end{array}$ & $\begin{array}{c}\text { Rendement } \\
(\%)\end{array}$ & $\begin{array}{c}\text { Poids vif } \\
\text { (g) }\end{array}$ & $\begin{array}{c}\text { Poids } \\
\text { carcasse (g) }\end{array}$ & $\begin{array}{c}\text { Rendement } \\
(\%)\end{array}$ & $\begin{array}{c}\text { Poids vif } \\
\text { (g) }\end{array}$ & $\begin{array}{c}\text { Poids } \\
\text { carcasse (g) }\end{array}$ & $\begin{array}{c}\text { Rendement } \\
(\%)\end{array}$ \\
\hline 16 & $\begin{array}{l}653,9 \text { a } \\
\pm 116,1\end{array}$ & $\begin{array}{l}495,0^{a} \\
\pm 108,1\end{array}$ & $\begin{array}{c}76,5^{\text {a }} \\
\pm 8,8\end{array}$ & $\begin{array}{r}640,9 \text { a } \\
\pm 114,9\end{array}$ & $\begin{array}{l}485,2 \text { a } \\
\pm 107,1\end{array}$ & $\begin{array}{l}74,9 \text { a } \\
\pm 8,6\end{array}$ & $\begin{array}{r}647,4^{\mathrm{a}} \\
\pm 115,5\end{array}$ & $\begin{array}{l}490,1^{a} \\
\pm 107,6\end{array}$ & $\begin{array}{c}75,7 \text { a } \\
\pm 8,7\end{array}$ \\
\hline 18 & $\begin{array}{l}780,0 \text { b } \\
\pm 159,1\end{array}$ & $\begin{array}{l}604,1 b \\
\pm 153,3\end{array}$ & $\begin{array}{l}78,8^{a} \\
\pm 7,6\end{array}$ & $\begin{array}{l}754,0 \text { b } \\
\pm 157,5\end{array}$ & $\begin{array}{l}583,9 b \\
\pm 151,7\end{array}$ & $\begin{array}{l}76,2^{a} \\
\pm 8,2\end{array}$ & $\begin{array}{l}767,0 \mathrm{~b} \\
\pm 158,3\end{array}$ & $\begin{array}{l}594,4 b \\
\pm 152,5\end{array}$ & $\begin{array}{l}77,5^{\text {a }} \\
\pm 7,9\end{array}$ \\
\hline 20 & $\begin{array}{l}886,9^{\text {c }} \\
\pm 164,1\end{array}$ & $\begin{array}{l}707,8^{\text {c }} \\
\pm 168,8\end{array}$ & $\begin{array}{l}81,6 \mathrm{~b} \\
\pm 9,9\end{array}$ & $\begin{array}{l}847,1^{\mathrm{c}} \\
\pm 162,5\end{array}$ & $\begin{array}{l}676,0^{c} \\
\pm 167,2\end{array}$ & $\begin{array}{l}78,0 \text { a } \\
\pm 7,3\end{array}$ & $\begin{array}{l}867,0^{c} \\
\pm 163,3\end{array}$ & $\begin{array}{r}691,9 \text { c } \\
\pm 168,0\end{array}$ & $\begin{array}{l}79,8 \mathrm{~b} \\
\pm 8,6\end{array}$ \\
\hline 22 & $\begin{array}{l}948,5^{d} \\
\pm 178,3\end{array}$ & $\begin{array}{l}799,6^{d} \\
\pm 196,2\end{array}$ & $\begin{array}{l}86,3^{c} \\
\pm 11,9\end{array}$ & $\begin{array}{l}905,5^{d} \\
\pm 176,5\end{array}$ & $\begin{array}{l}763,4^{d} \\
\pm 194,2\end{array}$ & $\begin{array}{l}82,3 b \\
\pm 10,9\end{array}$ & $\begin{array}{l}927,0^{d} \\
\pm 177,4\end{array}$ & $\begin{array}{l}781,5^{d} \\
\pm 195,2\end{array}$ & $\begin{array}{l}84,3^{\text {c }} \\
\pm 11,4\end{array}$ \\
\hline 56 & $\begin{array}{c}919,5^{\mathrm{e}} \\
\pm 176,36\end{array}$ & $\begin{array}{l}739,2 \mathrm{e} \\
\pm 188,4\end{array}$ & $\begin{array}{l}82,2 \mathrm{~b} \\
\pm 12,7\end{array}$ & $\begin{array}{l}878,1 \text { e } \\
\pm 174,8\end{array}$ & $\begin{array}{l}706,0 \text { e } \\
\pm 186,6\end{array}$ & $\begin{array}{l}78,6^{\text {a }} \\
\pm 11,7\end{array}$ & $\begin{array}{l}898,8^{\text {e }} \\
\pm 175,7\end{array}$ & $\begin{array}{l}722,6 \text { e } \\
\pm 187,5\end{array}$ & $\begin{array}{l}80,4^{b} \\
\pm 12,2\end{array}$ \\
\hline
\end{tabular}

Les chiffres présentant les mêmes lettres en exposant dans une colonne ne diffèrent pas significativement au seuil de $5 \%$ 
plus avantageux d'abattre tôt les oiseaux parce qu'il y a une baisse de rendement avec le temps.

L'augmentation de l'indice de consommation en fonction de l'âge a concordé avec les résultats obtenus lors d'autres études avec 2,6 au démarrage, 4 à la croissance et 6,5 à la finition $(3,9,25)$. L'indice moyen de consommation obtenu dans la présente étude, nettement plus élevé que ceux de 2,8,3,3 et 3,7 $(11,15,20)$, renforce l'observation d'Ayeni (2) qui note que la pintade locale est une mauvaise transformatrice d'aliments.

Les rendements carcasse et leur évolution ont été similaires à ceux observés au Nigeria (5). Le choix du rendement carcasse optimal pourrait intégrer les aspects organoleptique, nutritionnel, technologique et financier de la viande de la pintade. Ces différents aspects contribueront grandement au choix de l'âge optimal réel à l'abattage de la pintade locale selon le sexe et la variété.

\section{CONCLUSION}

Les résultats de cette étude confirment le caractère moyen du poids vif de la pintade locale du centre du Burkina Faso et sa faible aptitude à la valorisation des aliments. Ce poids vif pourrait toutefois être amélioré au regard de sa grande variabilité entre et dans les variétés étudiées. L'âge moyen optimal à l'abattage a été autour de 15 semaines pour les deux sexes. Ces différents âges restent toutefois à être confirmés par des études ultérieures qui intègreront les paramètres financiers et techniques de la qualité de la viande.

\section{BIBLIOGRAPHIE}

1. ASSOUMANE I., GOURO A.S., 1997. L'élevage des pintades au Niger. In: Sonaiya E.B., Ed., Proc. INFPD workshop Issues in family poultry research and development, Mbour, Senegal, 9-13 Dec. Ile-Ife, Nigeria, INFPD, p. 254-259.

2. AYENI J.S.O., 1983. Study of grey breasted guinea fowl (Numida meleagris galeata pallas) in Nigeria. World Poult. Sci. J., 39: 143-151.

3. AYORINDE K.L., 1987. Changes in anatomical points of the guinea hens in lay. Nig. J. Anim. Prod., 14: 121-123.

4. AYORINDE K.L., 2004. The spice of life. The seventy-first inaugural lecture. Ilorin, Nigeria, University of Ilorin, $60 \mathrm{p}$.

5. AYORINDE K.L., OLUYEMI I.A., AYENI J.S.O., 1988. Growth performance of four indigenous helmeted guinea fowl varieties (Numida meleagris galeata Pallas) in Nigeria. Bull. Anim. Health Prod. Afr., 36: 356-360.

6. BESSIN R., BELEM A.M.G., BOUSSINI H., COMPAORE Z., KABORET Y., DEMBELE M.A., 1998. Enquête sur les causes de mortalité des pintadeaux au Burkina Faso. Revue Elev. Méd. vét. Pays trop., 51 : 87-93.

7. BLUM J.C., GUILLAUME J., LECLERCQ B., 1975. Studies on the energy and protein requirement of growing guinea fowl. Br. Poult. Sci. 16: $157-168$

8. BONKOUNGOU G.F.X., 2005. Characteristics and performances of guinea fowl production under improved and scavenging condition in the Sahelian region of Burkina Faso. MSc Thesis, Royal Veterinary and Agricultural University, Copenhagen, Denmark, 59 p.

9. CARLIER S., 2000. Effet de la teneur des provendes en cellulose brute sur les performances pondérales des pintades et la qualité de leur viande en région chaude. Mémoire fin d'études, faculté de Sciences agronomiques, Gembloux, Belgique, 68 p.

10. CASTAING J., 1979. Aviculture et petits élevages. Paris, France, J.-B. Baillière, $313 \mathrm{p}$.

11. DEHOUX J.P., BULDGEN A., DACHET P., DIENG A., 1997. Influence de la saison et de la concentration énergétique de I'aliment sur les performances de croissance des pintadeaux (Numida meleagris) en région tropicale. Revue Elev. Méd. vét. Pays trop., 50 : 303-308.

12. DeMBELE P., GNOUMOU D., FREDERIC P., 1996. L'élevage de la pintade au Burkina Faso. Ouagadougou, Burkina Faso, Réseau documentaire élevage, 8 p. ( $\mathrm{n}^{\circ} 4$ spécial octobre)

13. DIABATE B., 1987. Etude de I'aviculture villageoise en zone Mali-Sud cas des villages de Djinigorola et Yaban. Mémoire Ingénieur Sciences appliquées, Institut polytechnique rural de Katibougou, Bamako, Mali, 48 p.

14. DIABATE H., 1981. Elevage traditionnel de la pintade en HauteVolta. Mémoire Ingénieur Développement rural, Institut supérieur polytechnique, Ouagadougou, Burkina Faso, 109 p.

15. FARKAS T., 1965. Interesting facts about the crowned guinea fowl (Numida meleagris). Fauna Flora, 16: 23-28.
16. JOHNSON A.S., GOWE R.S., 1962. Modification of the growth patterns of the domestic turkey by selection at two ages. In: Proc. 12th World's Poultry Cong., Sydney, Australia, p. 57-62.

17. KNIZETOVA H., HYANEK J., KNIZE B., ROUICEK J., 1991. Analysis of growth curve of guinea fowl. I. Chickens. Br. Poult. Sci., 22: 1039-1053.

18. KOEHL J.F., 1996. Enquête annuelle sur les coûts de production des volailles de chair. Paris, France, Institut technique de l'aviculture, p. 97-106.

19. LE COZ DOUIN J., 1992. L'élevage de la pintade. Maisons-Alfort, France, Le point vétérinaire, $252 \mathrm{p}$.

20. MACIEJOWSKI J., ZEIBA P., 1982. Genetics and animal breeding. Warsama, Poland, Polish Scientific publishers, $37 \mathrm{p}$

21. MIGNON-GRASTEAU S., BEAUMONT C., 2000. Les courbes de croissance chez les oiseaux. Prod. Anim., 13 : 337-348.

22. MINISTERE DES RESSOURCES ANIMALES, 1996. Quelques notions sur l'élevage de la pintade. Ouagadougou, Burkina Faso, ministère des Ressources animales, $26 \mathrm{p}$.

23. MINISTERE DES RESSOURCES ANIMALES, 2003. Méléagriculture au Burkina Faso : diagnostic et axes d'amélioration. Ouagadougou, Burkina Faso, ministère des Ressources animales, 46 p.

24. OGUNTONA T., 1982. Commercialising guinea fowl. West Africa. J. Anim. Prod., March/April: 52-54.

25. OGUNTONA T., 1983. Current knowledge of nutrients requirements of the gray breasted helmet guinea fowl. In: Ayeni J.S.O., Olomu J.M., Aire T.A., Eds, The helmet guinea fowl. New Bussa, Nigeria, Kainji Lake Research Institute, p. 121-128.

26. OUEDRAOGO H., 1987. Elevage de la pintade au Burkina Faso utilisation des termites, des asticots, de l'aliment complet dans le démarrage des jeunes. Mémoire fin d'études, Institut polytechnique rural de Katibougou, Bamako, Mali, 84 p.

27. SANFO R., 2005. Caractéristiques physiques de l'œuf de la pintade locale au centre du Burkina Faso. Rapport d'activités. Ouagadougou, Burkina Faso, Institut de l'environnement et des recherches agricoles, $33 \mathrm{p}$.

28. SAUNDERS M.J., 1984. Aviculture traditionnelle en Haute-Volta. Synthèse des connaissances actuelles et réflexions autour d'une expérience de développement, tome 1. Ouagadougou, Burkina Faso, Programme de développement des animaux villageois, $145 \mathrm{p}$.

29. SAVADOGO A., 1995. Contribution à l'amélioration de l'élevage de la pintade (Numida meleagris) au Burkina Faso. Mémoire fin d'études d'IDR, université d'Ouagadougou, Burkina Faso, 102 p.

30. SMETANA P., 1974. Some aspects of guinea fowl production. In: Proc. Australian Poultry Science Convention, Hobart, Tasmanea, Australia, p. 307-309.

Reçu le 21.04.2007, accepté le 11.09.2008 


\section{Summary}

Sanfo R., Boly H., Sawadogo L., Brian O. Local Guinea Fowl (Numida meleagris) Weight Performance under Improved Feeding System in the Central Region of Burkina Faso

The growth performance of 200 local guinea fowls (Numida meleagris) (97 females and 103 males) were studied in an improved feeding system. The hatching body weights were $25.8 \pm 3.1 \mathrm{~g}$ in females and $25.5 \pm 2.4 \mathrm{~g}$ in males. The body weights at inflexion points were $523.0 \pm 171.4 \mathrm{~g}$ in females and $497.0 \pm 134.7 \mathrm{~g}$ in males, and the asymptotic weights were $962.0 \pm 147.8 \mathrm{~g}$ and $857.0 \pm 183.7 \mathrm{~g}$, respectively. The weights at inflexion points and the asymptotic weights varied significantly $(p<0.05)$ depending on the variety. The daily weight gains at inflexion points were $10.1 \pm 4.7 \mathrm{~g}$ in females and $10.2 \pm 5.1 \mathrm{~g}$ in males, occurring at 12-14 weeks and 14-16 weeks of age, respectively, i.e. the optimum slaughtering age for each sex was within these intervals. The general average growth rate was $29.2 \pm 16.8 \%$ for both sexes $(30.0$ $\pm 18.0 \%$ in females and $28.4 \pm 15.9 \%$ in males). The carcass yield increased from $65.7 \%$ at 16 weeks to $74.3 \%$ at 22 weeks, and dropped to $70.4 \%$ at 56 weeks. The feed conversion ratio was 4.6 from 0 to 16 weeks in all the birds.

Keywords: Guinea fowl - Numida meleagris - Animal performance - Weight gain - Burkina Faso.

\section{Resumen}

Sanfo R., Boly H., Sawadogo L., Brian O. Rendimientos ponderales de la gallina de Guinea local (Numida meleagris) bajo un sistema de alimentación mejorada en la zona central de Burkina Faso

Se comunican los rendimientos de crecimiento ponderal de 200 gallinas de Guinea (97 hembras y 103 machos) locales (Numida meleagris), bajo un sistema de alimentación mejorada. Las hembras presentaron un peso vivo medio en el momento de la eclosión de 25,8 $\pm 3,1 \mathrm{~g}$ y los machos de 25,5 $\pm 2,4 \mathrm{~g}$. Los pesos vivos en los puntos de inflexión fueron de $523,0 \pm 171,4 \mathrm{~g}$ en las hembras y de $497,0 \pm 134,7 \mathrm{~g}$ en los machos y los pesos asintomáticos de $962,0 \pm 147,8$ g y $857,0 \pm$ $183,7 \mathrm{~g}$, respectivamente. Los pesos en los puntos de inflexión y los pesos asintomáticos difirieron significativamente $(p<0,05)$ según la variedad. Las ganancias de peso medio cotidianas en los puntos de inflexión fueron de 10,1 $\pm 4,7 \mathrm{~g}$ en las hembras y de 10,2 $\pm 5,1 \mathrm{~g}$ en los machos. Estos se adquirieron a intervalos de edad de 12 a 14 semanas y de 14 a 16 semanas respectivamente, edades óptimas para el sacrificio. El promedio general de las tasas de crecimiento fue de 29,2 $\pm 16,8 \%$ para los dos sexos, con $30,0 \pm 18,0 \%$ en las hembras y $28,4 \pm 15,9 \%$ en los machos. El rendimiento de la carcasa aumentó de $65,7 \%$ a 16 semanas a $74,3 \%$ a 22 semanas, para bajar a $70,4 \%$ a 56 semanas. El índice medio de consumo presentó un valor de 4,6 en el momento de la eclosión a 16 semanas de edad para los dos sexos.

Palabras clave: Gallina de guinea - Numida meleagris Desempeño animal - Ganancia de peso - Burkina Faso. 